\title{
DRIVERS FAIL TO CALIBRATE TO OPTIC FLOW SPEED CHANGES DURING AUTOMATED DRIVING
}

\author{
Callum Mole ${ }^{1}$, Gustav Markkula ${ }^{2}$, Oscar Giles ${ }^{1,2}$, Yuki Okafuji ${ }^{3}$, Richard Romano ${ }^{2}$, \\ Natasha Merat ${ }^{2}$, Richard Wilkie ${ }^{1}$ \\ ${ }^{1}$ School of Psychology, ${ }^{2}$ Institute of Transport Studies, University of Leeds, Leeds, UK \\ ${ }^{3}$ Department of Mechanical Engineering, Kobe University, Kobe, Japan \\ Email: c.d.mole@leeds.ac.uk
}

\begin{abstract}
Summary: The human perceptual-motor system remains well-calibrated during manual driving supporting successful steering despite changing conditions, such as alterations in vehicle speed. Automated vehicles may interrupt perceptual-motor calibration so that when a driver takes-over control they will not be prepared for the driving conditions. Optic flow is a powerful source of visual information for calibrating to speed changes during manual steering, but it is currently unclear whether humans are sensitive to changes in optic flow speed when they are not in active control of the vehicle (i.e. by relying upon vision alone). Here we used a driving simulator to examine sensitivity to changes in optic flow speed across active (manual steering) and passive (automated steering) modes of control. Optic flow speed was altered independent of vehicle speed. The mismatch between perceived speed and actual speed causes a well-calibrated motor system to be reliably biased. Drivers were asked to take-over manual steering control after a short $(\sim 10 \mathrm{~s})$ period of automation. Results showed that manual steering was not biased when flow speed was manipulated only in the automated period. One interpretation is that drivers had trouble recalibrating to optic flow changes that occurred during automated driving. If so, this suggests that there will exist a period where the perceptual-motor system is miscalibrated in the early stages of take-over after automated vehicle control.
\end{abstract}

\section{INTRODUCTION}

Over the course of a single car trip a driver may face a multitude of different conditions. Changes may be environmental (weather events that reduce road friction e.g. rain or ice), vehicular (e.g. increases in vehicle speed), or internal to the driver (e.g. muscle fatigue). Each change alters the required steering response, and specifically the relationship between how a perceptual variable (e.g. optic flow) is translated into a steering action: referred to henceforth as the perceptualmotor mapping. Under normal driving conditions perceptual-motor mappings need to be repeatedly and rapidly adjusted to ensure safe and efficient steering performance.

During manual control the perceptual-motor system copes well with these slight changes to steering conditions, effortlessly adapting the well-learned steering movements to maintain good perceptual-motor calibration to current task conditions (Fajen, 2005). There is considerable evidence from the perceptual-motor literature that humans can quickly adapt movements to a wide range of environmental alterations, providing there is the opportunity for active control (see Brand \& de Oliveira, 2017; Van Andel et al., 2017 for recent reviews). 
During highly automated driving, when conditions change the driver will not have access to the rich feedback provided by manual control of the vehicle. For example, a driver may have relinquished control at a slow vehicle speed before joining a motorway, so when a take-over request occurs (e.g. due to a failure in the AV system) the vehicle is travelling at higher speeds (see Mole et al., 2019 for a detailed discussion). Russell et al. (2016) examined this type of issue by simulating an increase in speed during automation by altering the steering ratio (how readily steering angle change is translated into road wheel angle change) independently of vehicle speed (which remained constant), effectively changing the perceptual-motor mapping. They observed that steering was unstable when drivers took-over control after the steering ratio change. Over successive trials drivers were able to bring steering performance back to baseline levels, but the process of recalibration happened over a period of 1-2 minutes.

In the Russell et al. (2016) experiment the perturbation happened to the vehicle during automation so that the change was not perceptible by participants until they took-over control. However, it is well known that humans are highly sensitive to optic flow information and readily use optic flow to control steering (e.g. Mole et al., 2016; Kountouriotis et al., 2016). It may be the case, then, that drivers are able to update perceptual-motor mappings to some extent through perceptually available changes in flow speed during automation, prior to manual control.

The current experiment examines whether drivers are sensitive to changes in flow speed during automation. In previous experiments it has been shown that artificially increasing or decreasing flow speed independently of vehicle speed produces reliable steering biases during manual control (Mole et al., 2016; Kountouriotis et al., 2016). In these experiments the perceptual-motor systems (that usually maintain good calibration) are effectively being hijacked: drivers calibrate to a new (but artificially biased) optic flow speed, causing steering errors. In other words, the steering biases are caused by the readiness of the perceptual-motor system to use optic flow to keep calibrated. In the current study we use these manipulations to probe calibration. If flow speed is manipulated only during automation (i.e. the flow speed bias is immediately removed upon take-over), and drivers are sensitive to these changes (i.e. they are able to update perceptual-motor mappings without active control of the vehicle), then they will show steering errors when taking over control. However, if drivers can only update the mapping if they are in active control than steering errors will be entirely dependent on whether flow speed is biased during manual control and manipulating optic flow during automation will not cause steering biases upon take-over.

\section{METHOD}

\section{Participants}

20 University of Leeds students and staff took part in this experiment (10 males \& 10 females, mean $=24.75 y r s$ ). All participants held driving licenses. Ethical approval was granted by the University of Leeds School of Psychology Research Ethics Committee (ref: 16-0381).

\section{Apparatus}

Stimuli were generated using Vizard 3.0 and back-projected onto a large projection screen (field of view $89^{\circ} \times 58^{\circ}$ ). Similar to Mole et al. (2016) the simulated stimuli consisted of a simple grass-textured ground-plane with super-imposed white lines for the road. Participants sat on a 
fixed-based height-adjustable driving seat, with eye-height measured at $1.2 \mathrm{~m}$ from the ground and $1 \mathrm{~m}$ away from the display.

\section{Transition Scenario}

The driver experienced many short trials of steering constant curvature bends of $60 \mathrm{~m}$ radius. Each trial began with a period of automation. The automated vehicle would begin in the center of the $3 \mathrm{~m}$ wide road, and slowly drift towards the outside edge, adopting a position with a lateral error of $1.25 \mathrm{~m}$ relative to the centerline. Drift was induced so that during manual control there were steering adjustments required to re-centre the trajectory in lane. The duration of the automation period was randomly selected from $10 \mathrm{~s}, 11 \mathrm{~s}$, or $12 \mathrm{~s}$ so that the point of takeover was unpredictable (but this was not a feature of interest so data analysis averages across these durations). The wheel motion was automated, but drivers had their hands-off the wheel so that they only had visual feedback available. Take-over was prompted by two separate tones sounding two seconds apart. At the first tone the participant returned their hands to the wheel. On the second tone the driver gained control of the vehicle. For the manual driving period drivers were tasked with bringing the vehicle back to the centre of the road. At the end of $10 \mathrm{~s}$ of manual driving a further tone sounded, prompting the driver to remove their hands from the wheel, the trial ended and the visual scene was reset (Figure 1A).

\section{Manipulating Flow Speed}

The locomotor speed was kept constant throughout all conditions, so during veridical conditions the movement of the ground texture (optic flow) and road-edges were consistent with driver motion. Some conditions artificially biased flow to increase or decrease only flow speed: on a constant curvature track, the speed can be altered independently of road edges by rotating the entire ground-plane around the curve origin (described in Mole et al., 2016), so it moves counter to the observer's direction of travel (increased perceived speed) or in the same direction (decreased perceived speed). Note that this manipulation primarily lengthens or shortens the longitudinal component of the ground texture flow, producing a sensation of driving faster or slower. Rotating the ground-texture does not alter the way that the visual scene responds to motion of the camera: a change in yaw will produce veridical reciprocal horizontal motion in both road-edges and ground-texture.

Mole et al. (2016) observed that, during manual control, participants exhibit oversteering (towards the inside road edge) when flow was faster-than-veridical, and understeering (towards the outside edge) when flow was slower-than-veridical (see also Kountouriotis et al., 2016). We follow Mole et al. (2016) and call this predictable difference in steering bias: Flow-InducedSteering-Bias (FISB). In our study FISB refers to steering biases present in the manual period (the trajectory taken by the automated vehicle did not vary across trials). 

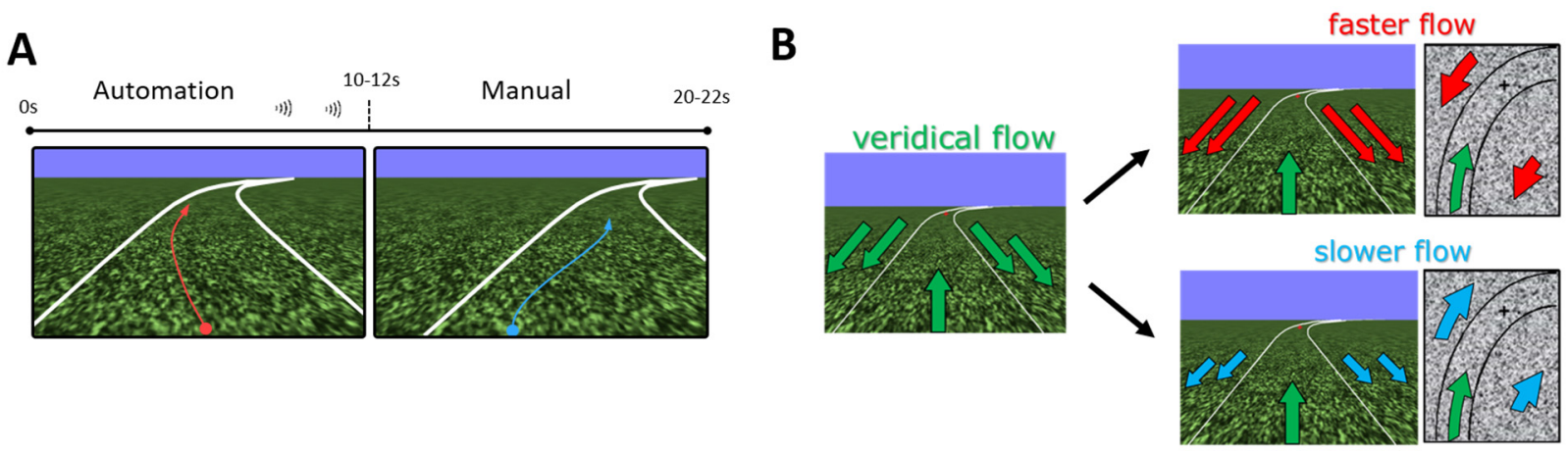

Figure 1. A) Trials consisted of approximately $10 \mathrm{~s}, 11 \mathrm{~s}$, or $12 \mathrm{~s}$ of automated driving, followed by $10 \mathrm{~s}$ of manual driving. During automation the vehicle drifted gradually to the outside of the bend. The driver is prompted regain a firm grip, and 2 s later to take over manual control of steering and return the vehicle to the road centre. B) A schematic of how the whole ground-plane can be rotated (top, fast) or counter-rotated (left) to increase or decrease optic flow speed whilst keeping locomotor velocity (green arrow) constant. Flow speed was altered by $50 \%$ of veridical speed.

Flow speed was manipulated either only during vehicle automation (Auto Only), only during manual control (Manual Only), or during both manual and automated control (Both). Based on Mole et al. (2016), we expected to observe FISB when the flow speed was manipulated when the driver was in manual control (Manual Only and Both). If drivers were sensitive to flow speed during automation, FISB should also be observed when the flow speed manipulation was present only in the automation period (Auto Only). Conversely, if drivers are only sensitive to flow speed during active control of steering then no FISB would be expected for Auto Only and instead FISB would only be observed for Manual Only and Both.

\section{Manipulating Gaze Fixation}

Eye-movements interact with optic flow information, so it was considered that a driver may be more sensitive to changes in flow speed if the location of gaze was similar across both manual and automation periods, compared to if there was a gaze shift. Therefore, three gaze fixation conditions were included. In two of these conditions the fixation placement was constant during both automation and manual: either placed at a 'Near' point, $3 \mathrm{~m}$ ahead (Near), or at a 'Far' point, $16 \mathrm{~m}$ ahead (Far). In a third condition the fixation placement was shifted: participants fixate the Near point during automation, then made a saccade to the Far point upon takeover (Near-Far) to approximate the type of gaze behavior likely to be observed in real-world settings.

\section{Experiment Design}

The combination of Flow Speed (Fast, Slow), Flow Presence (Auto Only, Manual Only, Both), and Gaze Fixation (Near, Far, Near-Far) resulted in 18 conditions. For each level of gaze fixation a baseline condition was included where flow was not manipulated, totaling to 21 conditions. These were randomly interleaved and there were 6 repeated trials for each condition. Before commencing the experiment, participants experienced 6 practice trials with veridical flow to familiarize themselves with the simulator and the task instructions. 


\section{RESULTS}

OverSteering Bias (in metres; OSB) was calculated to assess directional deviation from the roadcentre. Positive OSB indicates a road position towards the inside edge (oversteering), and negative OSB indicates a position towards the outside edge (understeering). Only the manual period was used to calculate OSB. Clear evidence of simulator error (described in the Supplementary Materials report on osf.io/yzgra) was found in nine out of the total 2,520 trials $(.36 \%)$, and these were removed. The average trajectories are shown in Figure 2.

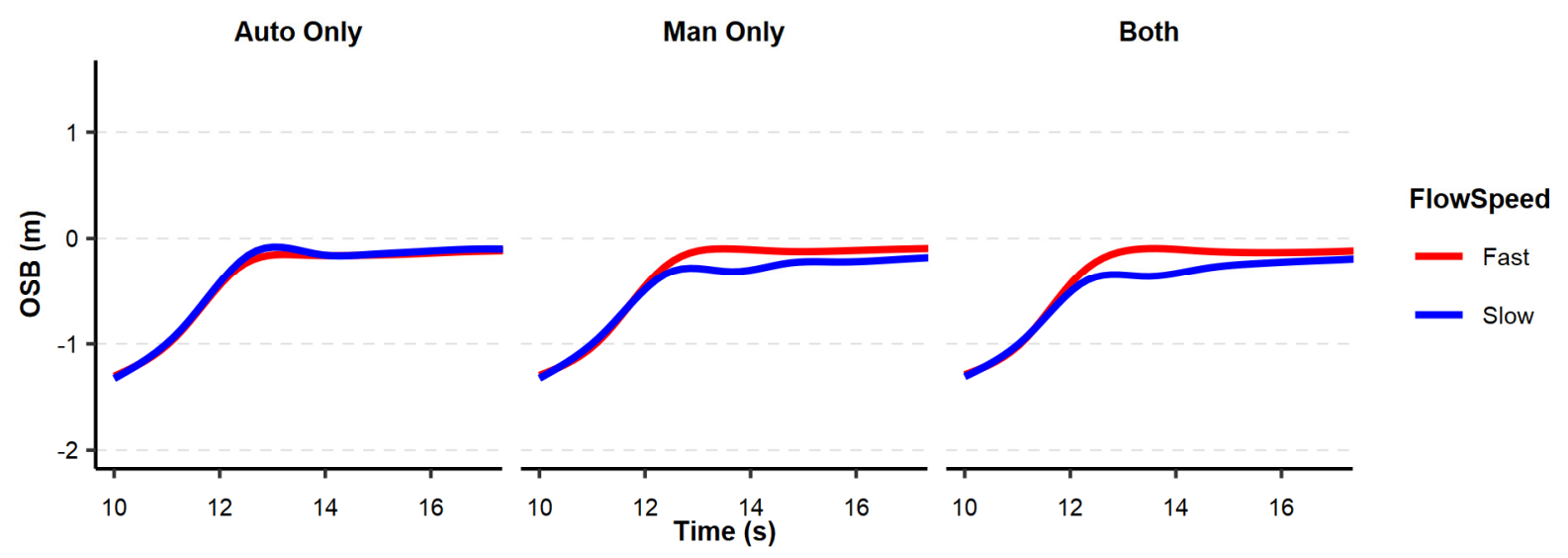

Figure 2. Average trajectories (collapsed across fixation condition) showing Oversteering Bias (OSB) over time for conditions where flow speed was increased (fast) or decreased (slow) during the automation period (Auto Only), the manual control period (Man Only), during both periods (Both).

\section{Model Specification}

A Bayesian estimation approach to inferential statistical analysis was adopted (McElreath, 2016), using a 3-factor hierarchical model that predicts steering bias. The factors are Flow Speed (Fast, Slow), Presence (Auto Only, Manual Only, Both), and Gaze Fixation (Near, Far, Near-Far). Coefficients are allowed to vary between participants. Posterior predictive checks showed that the model predictions align well with the raw data. Flow Presence and Gaze Fixation are explored in turn.

\section{Sensitivity of Flow Speed during Automation or Manual control}

Figure 2 appears to demonstrate that the effect of flow speed on steering bias interacts with whether the flow manipulation was present (Manual Only; Both) or was not present (Auto Only) during active control. Contrasts between Fast and Slow conditions were calculated to examine the probability of a reliable difference between flow speed conditions, shown in Figure 3A. For specific contrasts a useful heuristic for assessing whether a difference is reliable is whether the $95 \%$ highest density interval (HDI) excludes zero. There is $95 \%$ probability that an HDI contains the contrast mean. The contrast distribution for Both is centered away from zero, with a 95\% HDI that spans $.035 \mathrm{~m}$ to $.18 \mathrm{~m}$, and with $99.9 \%$ of the posterior mass above zero. The contrast for Manual Only is also centered away from zero (95\% HDI from .026 m to .16 6m, 99.5\% mass $>0$ ). However, the distribution for Auto Only is close to zero, with a 95\% HDI that encompasses zero $(-.075 \mathrm{~m}$ to $.022 \mathrm{~m}, 84.8 \%$ mass $<0)$. These contrast distributions clearly show an interaction: there is a high likelihood of a difference between Fast and Slow conditions in Both and Manual Only, but a low likelihood of a difference in Auto Only. 

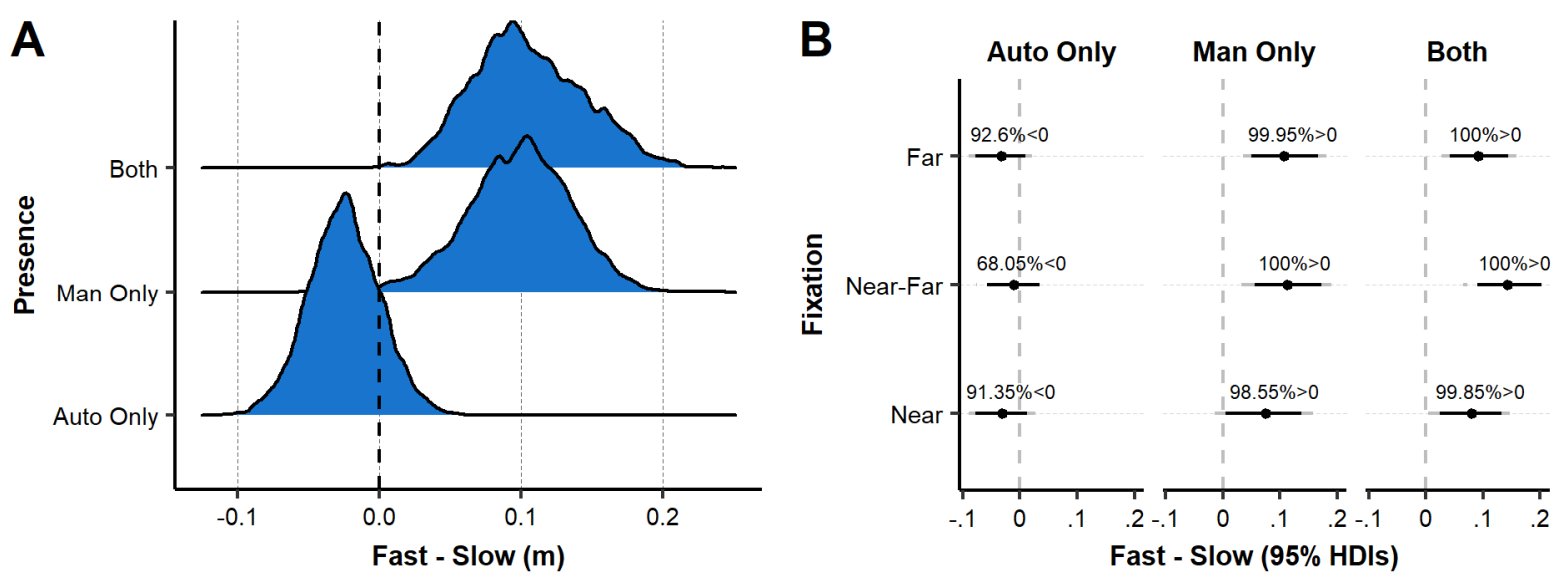

Figure 3. A) Posterior distributions for Flow-Induced Steering Bias (contrast between Fast and Slow flow speeds), for each presence manipulation. B) Posterior contrasts between oversteering bias in Fast and Slow flow speed conditions. The vertical dotted line corresponds to zero. The black point-ranges are $95 \%$ highest density intervals, and the grey point-ranges are $99 \%$ HDIs. A useful heuristic for assessing differences is that if more than $\mathbf{9 5 \%}$ of the probability distribution is greater than zero than it is a noteworthy difference.

There are hints in Figure 3A that the effect of flow speed may actually be reversed for Auto Only. To further examine this possibility contrast metrics for each condition were calculated (Figure 3B). The posterior distribution is clearly non-zero during Manual Only and Both, but the distribution during Auto Only is less clear, with the 95\% HDI encompassing zero in all cases. Whilst the peak is left of zero for all gaze fixation conditions, in practical terms the effect is very small $(\sim-0.025 \mathrm{~m})$.

\section{Role of Gaze Fixation in sensitivity to Flow Speed}

It can be seen in Figure 3B that the Gaze fixation conditions did not alter greatly the way that drivers responded to changes in flow speed. Although forcing individuals to look at a Near point did appear to increase understeer, this observation is not relevant to the core hypotheses.

\section{CONCLUSIONS}

In the current experiment drivers responded to changes in flow speed, but only if flow speed was manipulated during active control. One interpretation of the data could be that drivers were able to recalibrate very quickly (in the order of milliseconds) upon take-over in the Auto Only condition. However, previous experiments that manipulate flow speed show that error develops over a few seconds (see Figure 2, and also trajectories plotted in Mole et al., 2016), suggesting that recalibrating to flow speed is not immediate. More likely, drivers in the Auto Only condition did not calibrate to the altered flow speed during automation, so there was no recalibration needed when the flow speed was veridical after take-over. Whilst the absence of flow bias during Auto Only could be interpreted as a good thing (i.e. drivers did not show errors), the applied implications are potentially concerning: drivers may be unable to adapt to changes in vehicle speed that occur during periods of automation (when the driver is not in active control), so there is likely to be a period of unstable steering after take-over, where a driver recalibrates to new conditions (Russell et al., 2016;). Here, the flow speed was increased or decreased by 50\% and the locomotor speed was constant at $30 \mathrm{mph}$. This equates to a driver relinquishing control when 
travelling at $30 \mathrm{mph}$, having automated vehicle change speed to $45 \mathrm{mph}$ or $15 \mathrm{mph}$, and then the human driver taking back control without having adjusted to this new locomotor speed.

The principles explored in this experiment are not constrained to changes in speed. A change of environment could occur in almost any period of automation (even though vehicle speed may remain constant), and a driver would need to quickly update their control strategies to a new environment to remain safe. It remains to be seen whether (or how quickly) drivers can recalibrate to other changes in the driver-vehicle-environment, such as subtle changes in vehicle dynamics due to different surface textures, but our results suggest that active control of the vehicle is an essential component of safe recalibration. Therefore, take-over designs that support the driver during the potentially unsafe period of recalibration are recommended.

\section{ACKNOWLEDGMENTS}

This research was funded by EPSRC grant TRANSITION (EP/P017517/1). A preliminary analysis of this experiment was verbally presented at HFES Europe 2017 (Okafuji et al., 2017), and a non-peer reviewed brief report of this experiment has also been released on the Open Science Framework (osf.io/yzgra).

\section{REFERENCES}

Brand, M. T., \& de Oliveira, R. F. (2017). Recalibration in functional perceptual-motor tasks: A systematic review. Human movement science, 56, 54-70.

Fajen, B. R. (2005). Perceiving possibilities for action: On the necessity of calibration and perceptual learning for the visual guidance of action. Perception, 34(6), 717-740.

Kountouriotis, G. K., Mole, C. D., Merat, N., \& Wilkie, R. M. (2016). The need for speed: global optic flow speed influences steering. Royal Society open science, 3(5), 160096.

McElreath, R. (2016). Statistical Rethinking: A Bayesian Course with Examples in R and Stan. CRC Press.

Mole, C. D., Kountouriotis, G., Billington, J., \& Wilkie, R. M. (2016). Optic flow speed modulates guidance level control: New insights into two-level steering. Journal of Experimental Psychology: Human Perception and Performance, 42(11), 1818-1838

Mole, C. D., Lappi, O., Giles, O., Markkula, G., Mars, F., \& Wilkie, R. M. (2019). Getting back into the loop: the perceptual-motor determinants of successful transitions out of automated driving. Human factors, 0018720819829594.

Okafuji, Y., Mole, C., Wilkie, R. \& Merat, N. (Sept, 2017). Examining how driver steering behaviour is affected by optic flow after resuming control from a highly automated vehicle, The Human Factors and Ergonomics Society Europe Annual Meeting

Russell, H. E., Harbott, L. K., Nisky, I., Pan, S., Okamura, A. M., \& Gerdes, J. C. (2016). Motor learning affects car-to-driver handover in automated vehicles. Science Robotics, 1 (eaah5682).

van Andel, S., Cole, M. H., \& Pepping, G. J. (2017). A systematic review on perceptual-motor calibration to changes in action capabilities. Human movement science, 51, 59-71. 